\title{
ADOPCIÓN DEL COMERCIO ELECTRÓNICO EN EL TURISMO DE NIEVE Y DE MONTAÑ̃: ANÁLISIS DE LA PRESENCIA WEB DE LAS ESTACIONES DE ESQUÍ A TRAVÉS DEL MODELO EMICA
}

\author{
Natalia Daries-Ramon \\ Eduard Cristóbal-Fransi \\ Eva Martin-Fuentes \\ Estela Marine-Roig \\ Universidad de Lleida
}

\section{RESUMEN}

El papel de las Tecnologías de la Información y la Comunicación (TIC) en la gestión empresarial constituye actualmente un tema de creciente interés tanto para académicos como para profesionales. La Web 2.0 se ha revelado como una importante fuente de innovación que contribuye al desempeño organizacional y a la mejora de la competitividad de las empresas del sector turístico. A pesar de ello, se han encontrado evidencias que revelan las dificultades para la adopción de estas tecnologías en algunas empresas del sector turístico y su aplicación para un efectivo comercio electrónico. De acuerdo con lo anterior, el objetivo de este trabajo es analizar la implantación y uso de las herramientas de comercio electrónico en las estaciones de esquí de España y Andorra a través de la metodología eMICA (Extended Model of Internet Commerce Adoption), para identificar las oportunidades derivadas del uso de las herramientas Web 2.0 y mejorar las carencias detectadas para conseguir una gestión más competitiva.

Palabras clave: TIC; turismo; comercio electrónico; estación de esquí; modelo eMICA; Web 2.0; Turismo 2.0.

Recibido: 17 de marzo de 2015

Devuelto para su revisión: 14 de mayo de 2015

Aceptado: 15 de junio de 2015

Departamento de Administración de Empresas y Gestión Económica de los Recursos Naturales. Facultad de Derecho y Economía. Universidad de Lleida. C/Jaume II, 73. 25001 LLEIDA (España).E-mail: ndaries@aegern. udl.cat 


\title{
E-commerce adoption in mountain and snow tourism: analysis of ski resorts web presence through the eMICA model
}

\begin{abstract}
The role of Information and Communication Technologies (ICT) in business management is currently a topic of increasing interest to both academics and professionals. The Web 2.0 has emerged as an important source of innovation that contributes to organizational performance and improving the competitiveness of the tourism industry. However, evidence has been found that reveal the difficulties for the adoption of these technologies in the tourism industry and its application for effective e-commerce. Accordingly, the objective of this paper is to analyze the implementation and use of electronic commerce tools in the ski resorts of Spain and Andorra through the eMICA methodology (Extended Model of Internet Commerce Adoption) to identify opportunities arising from the use of Web 2.0 tools and improve the weaknesses identified for a more competitive management.
\end{abstract}

Keywords: ICT; tourism; electronic commerce; ski resorts; eMICA model; Web 2.0; Tourism 2.0.

\section{INTRODUCCIÓN}

Tal como comentan en su trabajo Buhalis y Law (2008), «el progreso tecnológico y el turismo han ido de la mano durante años». Está claro que la irrupción de las nuevas tecnologías en general y de Internet en particular ha influido en la forma de gestionar y de competir de las empresas (Porter, 2001). El sector turístico ha sido uno de los más afectados y donde se ha observado una adopción más generalizada de estas tecnologías en diversas áreas, pero particularmente en la manera en que las empresas turísticas distribuyen sus productos en el mercado (Buhalis, 2000; O'Connor y Frew, 2000; Leung et al., 2013). De hecho, tal como comentan en su trabajo DiPietro y Wang (2010), la adopción de las TIC por parte de las empresas turísticas influyen tanto en aspectos relacionados con la economía, como en la sociedad y la cultura. La aparición y el uso de nuevos dispositivos como smartphones o tabletas digitales y su influencia en el comportamiento de navegación en la Red y el comercio electrónico son muestra de ello.

En consecuencia y debido a este nuevo escenario, los consumidores utilizan cada vez más Internet como fuente de información sobre productos y servicios turísticos, como reservas hoteleras, alquiler de coches, vuelos, paquetes turísticos, etc., de la misma forma que está aumentando también la proporción de alojamientos y de viajes contratados a través de la Red (Litvin et al., 2008; Pérez Pérez et al., 2002; Xiang y Gretzel, 2010).

El uso de las TICs tiene una especial incidencia en la innovación en el sector turístico, dando lugar a la investigación y desarrollo de nuevos productos (Hjalager, 2010; Del Águila et al. 2010). Por otro lado, tenemos que tener en cuenta que la innovación no sólo se consigue incorporando tecnología, sino gestionándola correctamente. En este sentido, la aplicación del 
comercio electrónico al turismo permite la reducción de barreras geográficas, alcanzando por tanto una escala internacional y accediendo a más clientes (Ortega et al., 2012).

A nivel de comportamiento de los usuarios de servicios turísticos, los hábitos de compra y de consumo de los individuos también se han visto influenciados por las nuevas tecnologías (Kim y Fesenmaier, 2008; González-Rodrigo et al., 2010). De hecho, Internet permite que el consumidor sea cada vez más exigente y que esté mejor informado, y por ello asume cada vez más la gestión de sus viajes y desplazamientos (Law y Bai, 2008). Según Izquierdo et al., (2009), la Red proporciona a los usuarios de servicios turísticos una serie de ventajas como son la actualización rápida de la oferta, el acceso a los servidores, el ahorro de tiempo y la obtención de valoraciones de otros clientes entre otras. En este sentido, la adopción de las TICs e Internet por parte de los viajeros es cada vez mayor. El Instituto de Estudios Turísticos (2013) en su Informe Anual, señala que del total de los turistas que visitan España, el 64\% utiliza Internet para realizar alguna gestión referente a la planificación de sus vacaciones en nuestro país (ver tabla 1):

\section{Tabla 1}

\section{USO DE INTERNET POR PARTE DE LOS TURISTAS QUE VISITAN ESPAÑA}

\begin{tabular}{|l|c|l|c|}
\hline \multicolumn{2}{|c|}{ Servicios demandados } & \multicolumn{2}{c|}{ Ítems por servicio } \\
\hline \multirow{2}{*}{ Consulta } & \multirow{2}{*}{$64 \%$} & Transporte & $60 \%$ \\
& & Alojamiento & $48 \%$ \\
& & Actividades & $27 \%$ \\
\hline \multirow{3}{*}{ Reserva } & \multirow{2}{*}{$59 \%$} & Transporte & $56 \%$ \\
& & Alojamiento & $43 \%$ \\
& & Actividades & $11 \%$ \\
\hline \multirow{3}{*}{ Pago } & \multirow{2}{*}{$56 \%$} & Transporte & $55 \%$ \\
& & Alojamiento & $34 \%$ \\
\hline
\end{tabular}

Fuente: IET (2013). Encuesta de Gasto Turístico (Egatur). Informe Anual. Documento disponible en http://www. iet.tourspain.es

Las redes sociales, apoyadas en las capacidades de la Web 2.0, han revolucionado la comunicación y el comercio en turismo y viajes (Xiang y Gretzel, 2010). En relación al uso de las redes sociales, Cristóbal et al. (2013) afirman que tienen un impacto considerable en el sector de los hoteles, campings y agencias de viaje respecto a otros sectores. El principal uso que dan las pymes y grandes empresas turísticas a las redes sociales es su integración en las estrategias de marketing y el contacto e información con los clientes. Por otro lado, las microempresas van por delante en el uso de estas herramientas como canal de comunicación entre empleados/profesionales. Sin embargo, el uso de las redes sociales para la búsqueda y/o selección de personal tiene una incidencia menor, especialmente en microempresas.

Los trabajos realizados por Chung y Buhalis (2008) y Tsimonis y Dimitriadis (2014) también señalan la importancia que tienen en el consumo turístico el uso de las redes sociales 
y las comunidades online. Los resultados que obtienen muestran que las empresas que desarrollan comunidades online generan mayores beneficios y posibilidades de negocio además de una mayor fidelidad de los consumidores. De hecho, la interactividad de las empresas con los clientes a través de redes sociales, se ha convertido en una herramienta fundamental para crear vínculos con los clientes, aumentar su satisfacción e implicación, aumentar el tiempo de navegación, mejorar la imagen de marca y tener mayores oportunidades para la distribución del producto (Huertas y Marine-Roig, 2015). Asimismo, del estudio de Chung y Buhalis (2008) se desprende que la mayoría de los usuarios de estas comunidades las perciben como útiles ya que satisfacen sus necesidades de información, gracias a las opiniones, recomendaciones, comparativas que se pueden encontrar. No obstante, todavía queda mucho camino por recorrer y es necesario mejorar las relaciones y la comunicación entre los organismos públicos y las empresas privadas. Se constata, que en general, no hay interacción entre las redes sociales oficiales de los destinos españoles y las de las empresas privadas del sector turístico que es esencial para la gestión conjunta del destino turístico (Daries et al., 2014).

Una vez vista la significativa influencia de las nuevas tecnologías en la gestión de las empresas turísticas, y en el comportamiento de los consumidores de productos y servicios turísticos, consideramos necesario determinar el grado de adopción del comercio electrónico en un sector tan importante como el turismo de nieve, a través del análisis de la presencia online de las estaciones de esquí. Por esto, el principal objetivo de este estudio es aplicar la metodología basada en el modelo eMICA al caso de estudio de las estaciones de esquí españolas pertenecientes a la asociación ATUDEM (Asociación Turística de Estaciones de Esquí y Montaña) y a las de Andorra.

\section{TURISMO DE NIEVE}

El turismo de nieve lo podemos englobar dentro del turismo deportivo y del turismo de naturaleza, ya que esta disciplina se genera motivada por el deseo de practicar actividades deportivas en un entorno natural, de nieve y montaña (Esteban, 1995). Por otro lado Saz y Carús (2008), acuñan el concepto de Segmento Turístico Recreativo de Alta Montaña (STRAM) y lo definen como «el formado por Centros Turísticos Recreativos de Alta Montaña (CTRAM) o destinos que a través de una inversión previa en recursos humanos, equipos e infraestructura diversa explotan en ubicaciones de alta montaña, también fuera de la época invernal, centros recreativos o de ocio» (pp. 19-20).

A nivel territorial, las actividades turísticas, especialmente las relacionadas con el esquí han sido ampliamente promovidas desde los años 50 en las zonas montañosas españolas, como los Pirineos, para el desarrollo económico de las mismas (Lasanta et al., 2007). La oferta española de turismo de nieve y de montaña representa un total de 1.039 kilómetros de pistas esquiables, una cifra que llama la atención al compararse con los $3.168 \mathrm{~km}$ de costa española. Para contextualizar el sector, es interesante visionar el mapa topográfico de la península ibérica elaborado por ATUDEM, en el que se aprecia la distribución espacial de las diferentes estaciones, en todas las cordilleras de la península con elevación considerable sobre el nivel del mar. Así vemos que las estaciones de esquí son una realidad que afecta a un gran número de CC.AA. (Andalucía, Aragón, Asturias, Cantabria, CastillaLeón, Cataluña, Madrid, Galicia, La Rioja y Navarra). 


\section{Figura 1 \\ MAPA DE LAS ESTACIONES DE ESQUÍ EN ESPAÑA}

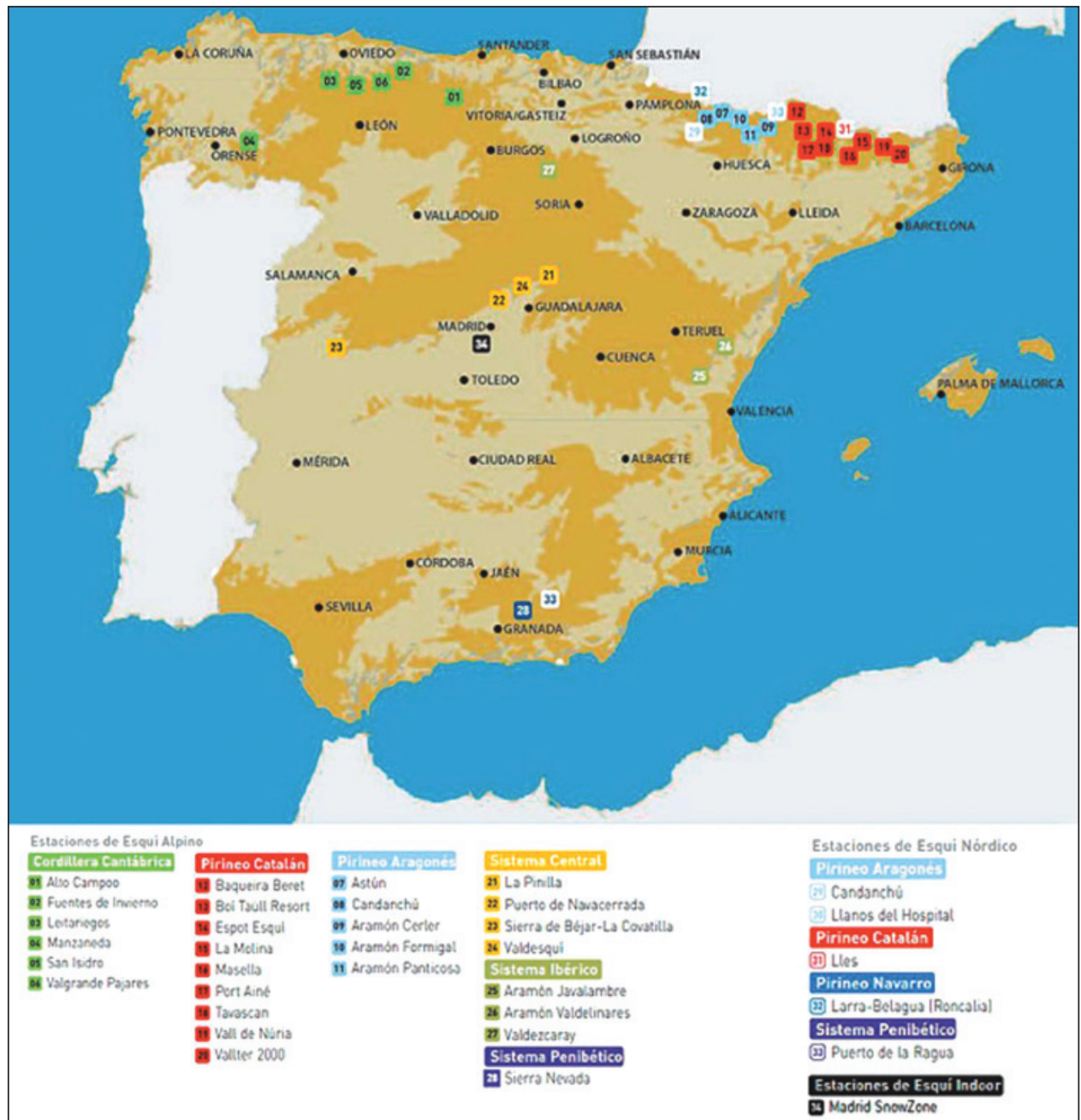

Fuente: ATUDEM (2015). Mapa de ubicación de las estaciones de esquí españolas. Documento disponible en: http://atudem.org/estaciones/mapa-de-ubicacion.html

La mayoría de las estaciones de esquí se circunscriben en la modalidad de estaciones de esquí alpino, pero un gran número de las estaciones enumeradas en este mapa, son a su vez estaciones de esquí nórdico. El esquí alpino es una modalidad más conocida y moderna que consiste en descender por una montaña nevada mientras que el esquí nórdico consiste en desplazarse de un sitio a otro.

En el sector de las estaciones de esquí, las de esquí nórdico suponen un porcentaje significativamente inferior tanto a nivel numérico como económico. A pesar de ello, 
todas ellas forman parte de los recursos turísticos de las zonas donde se ubican y tienen una clara utilidad como modo de aproximación a los deportes de invierno. En conjunto, las estaciones de esquí españolas representan un sector económico en alza que, en los últimos diez años, ha destinado más de 700 millones de euros a la inversión en mejoras de sus estaciones y cuenta con un creciente número de deportistas y turistas que visitan las estaciones o que realizan jornadas de esquí. Este sector desarrolla a su vez actividades económicas colaterales como transporte, hostelería, comercio de ropa y complementos, alquiler y venta de material deportivo, etc. (García Abad, 2012).

Según datos de ATUDEM (2015), el número de visitantes a las estaciones de esquí, superan los cinco millones de personas y ofrecen una oferta de alojamiento de más de 217.000 plazas. Por tanto, los datos que documentan el flujo de turistas hacia las zonas de montaña ponen de manifiesto que el sector ha cobrado una importancia económica determinante (Saz y Carús, 2008).

Tabla 2

CIFRAS EN LAS ESTACIONES DE ESQUÍ ALPINO

\begin{tabular}{|l|c|c|c|c|c|c|c|c|c|}
\hline Temporada & $\mathbf{2 0 0 4 - 2 0 0 5}$ & $\mathbf{2 0 0 5 - 2 0 0 6}$ & $\mathbf{2 0 0 6 - 2 0 0 7}$ & $\mathbf{2 0 0 7 - 2 0 0 8}$ & $\mathbf{2 0 0 8 - 2 0 0 9}$ & $\mathbf{2 0 0 9 - 2 0 1 0}$ & $\mathbf{2 0 1 0 - 2 0 1 1}$ & $\mathbf{2 0 1 1 - 2 0 1 2}$ & $\mathbf{2 0 1 2 - 2 0 1 3}$ \\
\hline Número de visitantes & 6.254 .715 & 7.165 .109 & 4.559 .144 & 5.160 .195 & 6.657 .778 & 5.618 .059 & 5.541 .159 & 4.505 .715 & 5.110 .000 \\
\hline $\begin{array}{l}\text { Capacidad total de transporte } \\
\text { (persona/hora) }\end{array}$ & 384.858 & 392.182 & 421.660 & 459.747 & 467.677 & 456.748 & 454.852 & 449.457 & 460.300 \\
\hline Pistas Balizadas (km) & 887 & 888 & 953 & 332 & 1.036 & $1.007,42$ & $1.011,96$ & $1.026,82$ & $1.039,50$ \\
\hline Producción nieve artificial (km) & 274 & 292 & 321 & 333 & 358 & 339 & 349 & 365 & 375 \\
\hline Oferta alojamiento (1) & 133.153 & 163.996 & 174.138 & 176.482 & 220.026 & 216.452 & 213.699 & 240.911 & 217.400 \\
\hline Inversiones (miles de euros) & 11.845 & 73.056 & 99.510 .291 & 72.596 .173 & 50.365 .331 & 54.291 .895 & 59.724 .303 & 24.372 .277 & - \\
\hline
\end{tabular}

(1) Hoteles, apartamentos, albergues y casas rurales, incluida área de influencia (radio $=35 \mathrm{Km}$.) Fuente: elaboración propia a partir de ATUDEM (2015)

Siguiendo el trabajo de Falk (2010), la demanda de turismo de invierno depende de diversos factores: la renta nacional e internacional, los precios, el transporte, los costes, la ubicación de las vacaciones de Pascua y el cambio climático. El cambio climático se revela como un factor importante, sobre todo para algunas estaciones de esquí de baja altura. En estas estaciones de esquí, el descenso de la demanda es mucho más pronunciada, ya que son muy dependientes del grosor de la nieve (Falk, 2010). De esta manera, las empresas invierten sobre todo en estaciones de esquí de altura con el objetivo de crear grandes dominios esquiables (Gerbaux y Marcelpoil, 2006; Falk, 2009) para atraer a esquiadores extranjeros (franceses, ingleses y rusos).

Por tanto, las estaciones de esquí de nuestro país se ven afectadas por nuevos competidores de Europa del Este que ofrecen nuevas infraestructuras y precios atractivos. Los gerentes de las estaciones de esquí españolas deben enfrentarse al envejecimiento de sus instalaciones. De hecho, un dominio esquiable se caracteriza por la infraestructura técnica para la práctica del esquí, esto es: pistas de esquí, senderos, aseos, equipos de nieve artificial, taquillas e instalaciones de recepción. (Gonçalves, 2012) 
En esta línea Pearce (2005) propone que en la decisión de viajar a una estación de esquí en invierno intervienen tanto aspectos internos como las características del individuo y los motivos personales y aspectos externos como son las características del destino. Por un lado el viajero desea un conjunto de actividades que le permite obtener una serie de experiencias deseadas. Por lo tanto, las actividades disponibles afectan a la elección de destino. Las motivaciones del viajero y la capacidad percibida de un destino para satisfacer las expectativas previas son variables estratégicas en el marketing de destinos (Mätzler y Siller, 2003). Por consiguiente, la manera de comunicar las actividades que se realizan en las estaciones de esquí es clave a la hora de la elección del destino.

\section{EL MODELO EMICA}

El Modelo de Adopción del Comercio por Internet (MICA) fue desarrollado originalmente para una investigación aplicada a la industria de fabricación de metal en Australia (Burgess y Cooper 1998). El modelo propone que en el desarrollo de sitios Web comerciales, las pymes suelen iniciarse en Internet a través de una simple página Web, y que esta presencia se hace más compleja con el tiempo, incorporándose nuevos procesos, ya que su experiencia y conocimientos en el uso de las TIC aumenta. MICA consta de tres etapas, incorporando tres niveles de procesos de negocio: a) la promoción basada en la Web; b) el suministro de información y servicios; y c) el proceso de transacciones. Las etapas de desarrollo proporcionan una hoja de ruta que indica en qué nivel se ubica un sector de negocio en su desarrollo de aplicaciones de comercio electrónico. En nuestro caso, las estaciones de esquí.

Como los sitios Web evolucionan continuamente, este movimiento se refleja a través de las diferentes etapas de desarrollo desde su inicio (promoción), a través de la consolidación (provisión) y finalmente la madurez (procesamiento). En el modelo se añaden niveles de complejidad y funcionalidad del sitio Web. Esta incorporación de niveles refleja la evolución de la empresa al pasar de una presencia en Internet estática a un sitio Web dinámico a través de los crecientes niveles de interactividad que incorpora integración de la cadena de valor y aplicaciones innovadoras para agregar valor a través de la gestión de la información y una mayor funcionalidad (Burgess et al., 2005).

Posteriormente, el modelo MICA se ha aplicado a diferentes sectores, entre ellos en la industria del turismo (Burgess y Cooper 2000; Doolin et al., 2002; Burgess et al., 2011; Ting et al., 2013), lo que ha permitido mejorar el modelo de partida con un modelo extendido de Adopción del Comercio por Internet (eMICA), en donde se agregan varias capas de sofisticación y se adapta el modelo MICA al nuevo entorno virtual, y a las particularidades de la Red (ver tabla 3). Este modelo basado en una evaluación por fases permite evaluar los sitios Web desde un nivel promocional hasta los procesos transaccionales que constituyen la fase superior de estadio en un sitio Web, pero cuenta con algunas limitaciones. Schmidt (2006) señala que tienden a reducir el nivel de complejidad de investigaciones, y que un sitio Web puede estar en dos niveles de desarrollo al mismo tiempo o en ninguno de ellos. Nuestro objetivo es, realizada la adaptación del modelo, comprobar el grado de madurez de los sitios Web de las estaciones de esquí, y en este sentido, el modelo eMICA, recoge las dimensiones lógicas que un sitio en Internet, como plataforma tecnológica, posee: información, comunicación y transacción (Marimon et al., 2010). 


\section{Tabla 3 \\ MODELO AMPLIADO DE ADOPCIÓN DEL COMERCIO ELECTRÓNICO}

\begin{tabular}{|c|c|c|}
\hline eMICA & & Ejemplos de funcionalidad \\
\hline \multirow{3}{*}{ Fase 1} & Promoción & \\
\hline & $\begin{array}{l}\text { Nivel } 1 \text { Información } \\
\text { básica }\end{array}$ & $\begin{array}{l}\text { Denominación, dirección física y detalles de con- } \\
\text { tacto, estado de la estación, estado de los accesos } \\
\text { a la estación. }\end{array}$ \\
\hline & Nivel 2 Información rica & $\begin{array}{l}\text { Informe anual, contactos de correo electrónico, } \\
\text { información sobre las actividades y entorno de } \\
\text { la empresa, e incentivos a través de Internet, parte } \\
\text { meteorológico. }\end{array}$ \\
\hline \multirow{4}{*}{ Fase 2} & Provisión & \\
\hline & $\begin{array}{l}\text { Nivel } 1 \text { Interactividad } \\
\text { baja }\end{array}$ & $\begin{array}{l}\text { Catálogo básico del producto, hipervínculos a } \\
\text { otras informaciones, formulario de consulta onli- } \\
\text { ne, posibilidad de rellenar encuestas en línea. }\end{array}$ \\
\hline & $\begin{array}{l}\text { Nivel } 2 \text { Interactividad } \\
\text { media }\end{array}$ & $\begin{array}{l}\text { Catálogos completos del producto, soporte al } \\
\text { usuario (FAQs, mapas del sitio Web, tour virtua- } \\
\text { les, georreferenciación, Webcam, etc.), informa- } \\
\text { ción del sector. }\end{array}$ \\
\hline & $\begin{array}{l}\text { Nivel } 3 \text { Interactividad } \\
\text { alta }\end{array}$ & $\begin{array}{l}\text { Chat, foros de discusión, multimedia, newsletters } \\
\text { o noticias por e-mail. Presencia en las redes so- } \\
\text { ciales y enlaces a sitios Web de valoración de } \\
\text { servicios turísticos. }\end{array}$ \\
\hline Fase 3 & Proceso & $\begin{array}{l}\text { Transacciones seguras, firma digital y encrip- } \\
\text { tación, estado y seguimiento de pedidos, inte- } \\
\text { racción con servidores y bases de datos, Web 2.0, } \\
\text { contenido generado por usuarios. }\end{array}$ \\
\hline
\end{tabular}

Fuente: Burgess et al. (2011) a partir de Burgess y Cooper (2000).

A pesar de que las empresas del ámbito turístico han sido pioneras en el uso intensivo de los recursos digitales, todavía quedan ciertos sectores que aún no aprovechan todas las posibilidades derivadas de las TIC (Álvarez et al., 2013). En nuestro caso, los escasos estudios realizados indican que los sitios Web de las estaciones de esquí no incorporan funciones avanzadas para el mantenimiento de las relaciones con sus clientes, utilizando sus sitios Web como herramientas de comunicación, obviando la posibilidad de la interactividad y de comunicación uno a uno. 


\section{METODOLOGÍA}

Dada la importancia económica del turismo de nieve (Lasanta et al., 2007), la aparición de nuevos mercados competidores y los beneficios que consideramos que supone la adopción de las nuevas tecnologías para mejorar la gestión de las estaciones de esquí. El principal objetivo del presente trabajo es analizar la implantación y uso de las herramientas de comercio electrónico en las estaciones de esquí de España y Andorra a través de la metodología eMICA (Extended Model of Internet Commerce Adoption) planteado por los autores Burgess y Cooper, (2000). Consiste en posicionar el sitio en función de 3 etapas: «promoción», que recoge informaciones acerca de la empresa; la segunda etapa es «provisión» que indica el nivel de interactividad; y la tercera es «procesamiento» que muestra el nivel de transacciones.

De esta forma se realizó la categorización de cada sitio según las fases y niveles propuestos por el modelo eMICA. Es necesario señalar que debido a que el modelo eMICA responde a un proceso de adopción gradual de Internet, es posible encontrar casos en los cuales los sitios reúnan funcionalidades e incorporen elementos propios de diferentes etapas y niveles del modelo. En estos casos, el posicionamiento otorgado es del más alto nivel, aun cuando pueda existir ausencia de algún ítem perteneciente a los niveles o etapas básicas del modelo. Adicionalmente se realizó una modificación en el modelo eMICA ya que se incorporaron algunas funcionalidades que no habían sido consideradas en el modelo original

A partir de los resultados obtenidos se busca:

- Proponer un modelo integral para evaluar sitios Web de estaciones de esquí en base a categorías de contenido y al nivel de madurez técnica observada (etapas de desarrollo o profundidad).

- Utilizar el modelo propuesto para evaluar los sitios Web de la totalidad de las estaciones de esquí en España y Andorra.

- Proporcionar recomendaciones y sugerencias para una gestión más eficiente de la presencia online de las estaciones de esquí.

Para alcanzar los objetivos propuestos se analizaron los sitios Web correspondientes a la totalidad de las estaciones de esquí y montaña asociadas a ATUDEM: 31 Alpinas, 21 Nórdicas y 1 Indoor. El estudio se extiende a su vez a los dominios esquiables de Andorra, debido a la gran tradición en el deporte blanco que posee el principado de Andorra y la gran afluencia de esquiadores de nuestro país. En total han sido 53 sitios Web analizados. Las observaciones fueron realizadas durante los meses de enero a abril de 2015.

Las variables utilizadas para evaluar el nivel de adopción del comercio electrónico en las Webs de las estaciones de esquí fueron adaptadas del modelo eMICA y seleccionadas a través de la revisión de la literatura. A su vez se ha determinado que para pasar de un nivel a otro y consolidar su posición, la Web tiene recoger un número mínimo de atributos (García-Lastra y Escalera, 2009; Álvarez, 2014). Por tanto, se pasará de nivel siempre que el sitio Web contenga las variables correspondientes al último nivel o capa. La escala es dicotómica para todas las variables. 


\subsection{Primera Fase: Promoción (información)}

Esta primera fase se centra en el uso de la Web como instrumento de comunicación de la estación de esquí y de sus productos al mercado. Esta fase está caracterizada por un escaso nivel de funcionalidad, contenido básico de carácter visual e informativo. Presenta dos niveles: en el primer nivel se exige tener un mínimo de 3 variables de las 6 propuestas y en el segundo también se necesitan 3 variables sobre las 7 posibles.

Tabla 4

VARIABLES DE LA PRIMERA FASE

\section{FASE 1: PROMOCIÓN (INFORMACIÓN)}

Nivel 1: Información Básica (mínimo de 3 variables de las 6 propuestas.)

Datos de contacto: Denominación, dirección, teléfono, fax, otros

Fecha y Hora de actualización

Estado de la estación: Abierta / Cerrada

Accesos a la estación: Abiertos / Cerrados / Cadenas

Fotos de la estación

Información de la localización de la estación

Nivel 2: Información Abundante (mínimo de 3 variables de las 7 propuestas)

E-mail y/o Formulario de contacto

Informe de pistas: Perfil, remontes, espesores, cotas, otros

Parte de meteorología: Actual / Previsión

Página Web disponible en más de un idioma.

Certificaciones de calidad

Comunicación de noticias / eventos

Promociones e incentivos a través de Internet (bonos / cupones, ofertas sólo por

Internet, concursos online)

Fuente: elaboración propia a partir del modelo eMICA (Burgess et al., 2011).

\subsection{Segunda Fase: Provisión (información dinámica)}

En esta segunda fase se determina si el sitio Web ofrece información dinámica de la estación y de su entorno. Y este último aspecto es clave ya que una estación de esquí y montaña se define como un destino turístico, o sea, como un espacio geográfico, con rasgos propios y capacidad para desarrollar instrumentos comunes de planificación que se comercializa con carácter integral (García-Lastra y Escalera, 2008). Esta fase presenta 
Tabla 5

VARIABLES DE LA SEGUNDA FASE

\section{FASE 2 : PROVISIÓN (INFORMACIÓN DINÁMICA) \\ Nivel 1: Nivel bajo de interactividad (mínimo de 4 variables de las 9 propuestas)}

Tarifas forfaits y abonos temporada

Plano de pistas

Enlaces información interna: Alojamientos, restaurantes, otros

Enlaces información externa: Alojamientos, restaurantes, otros

Enlaces a servicios de la estación: (escuela, guardería, guardaesquíes)

Información de la estación en Invierno - Verano (enlaces)

Calendario completo de la temporada

Reglamento seguridad en pistas

Reglamento condiciones uso forfait

Nivel 2: Nivel medio de interactividad (mínimo de 6 variables de las 12 propuestas)

Mapa Web

Web cam

Posibilidad de hacer reservas de alojamiento

Posibilidad de hacer reservas de forfait (sólo forfait)

Descarga de folletos y/o materiales y/o fotos

Envío de noticias por e-mail

Política de privacidad o aviso legal

Encuestas en línea

FAQs

Sugerencias

Buscador (por palabras, etc.)

Tienda online (como escaparate)

Nivel 3: Nivel alto de interactividad (mínimo de 6 variables de las 13 propuestas)

Zona Clientes / Socios

Plano de pistas interactivo

Aplicaciones multimedia

Blogs, Foros y/o Chats

Boletines por email (Newsletter)

Acceso a los perfiles en redes sociales de la estación de esquí

Posibilidad de recoger comentarios en línea de los clientes.

Posibilidad a los clientes de votar sobre la calidad /satisfacción con servicios prestados.

Enlaces a Webs de valoración de servicios turísticos (Tripadvisor, etc)

Tour Virtual

Vídeos o animación flash

Versión Web móvil

Descarga de app para móvil

Fuente: elaboración propia a partir del modelo eMICA (Burgess et al., 2011) 
tres niveles. Para superar el primer nivel se requiere un mínimo de 4 variables de las 9 propuestas. Para el segundo nivel se necesita disponer de 6 de las 12 variables propuestas y para el tercer nivel, se exigen 6 variables de las 13 disponibles.

\subsection{Tercera Fase: Proceso (Madurez Funcional)}

En la tercera fase, la presencia en Internet para la estación de esquí se convierte en un potente medio de comercio electrónico. Permite realizar la venta de los productos y/o servicios ofrecidos, a través de la Red combinándolo con los canales tradicionales. Recoge el grado de madurez funcional del sitio. Para estar en esta fase se necesita un mínimo de 2 variables de las 5 propuestas.

\section{Tabla 6 \\ VARIABLES DE LA TERCERA FASE}

\begin{tabular}{|l|}
\hline $\begin{array}{l}\text { FASE 3: PROCESO (MADUREZ FUNCIONAL) (mínimo de } 2 \text { variable de las } 5 \\
\text { propuestas) }\end{array}$ \\
\hline Proceso de compra completo (recarga) forfait \\
\hline Proceso de compra completo tienda online (otros productos) \\
\hline Proceso de compra completo alojamiento \\
\hline $\begin{array}{l}\text { Transacción online segura (en los procesos de compra posibles, firma digital, } \\
\text { encriptación, código de seguridad vía móvil) }\end{array}$ \\
\hline $\begin{array}{l}\text { Interacción con el servidor: consulta de la base de datos (acceso a tu perfil de cliente y } \\
\text { posibilidad de modificación, acceso al historial de compras. etc.) }\end{array}$ \\
\hline
\end{tabular}

Fuente: elaboración propia a partir del modelo eMICA (Burgess et al., 2011).

\section{ANÁLISIS DE RESULTADOS}

La información contenida en este apartado constituye un indicador acerca de la situación en la que se encuentra el sector del turismo de nieve respecto a las aplicaciones comerciales de Internet. Si analizamos los resultados en su totalidad, estos muestran que todavía queda margen de mejora en la adopción del comercio electrónico por parte de las estaciones de esquí y montaña en la península ibérica. El hecho de que únicamente el 15,1\% de los sitios Web hayan alcanzado la fase 3, y que la gran mayoría se sitúe en el nivel 2 de la segunda fase $(60,4 \%)$, indica el escaso grado de madurez funcional alcanzado por las estaciones en el desarrollo de sus sitios Web. Esto es indicativo de que se ha producido un cierto desplazamiento desde una perspectiva estática a una Web dinámica y con crecientes niveles de interactividad. No obstante cabría apuntar que independientemente de que los resultados revelan que casi el 68\% de los sitios Web presentan interactividad media, en gran medida este número está representado por las estaciones que poseen descarga de material u opción a suscripciones, con una menor representatividad de herramientas Web 2.0 o tienda online. 
Respecto a la fase 3 de Proceso que agrupa al $15 \%$ de las Webs evaluadas, se puede observar que la mayor parte de las Web además de ofrecer procesos de compra y pasarelas de pago seguro proporcionan mecanismos de comunicación que favorecen la interactividad con el usuario. No obstante, que sólo el $49 \%$ de las estaciones objeto de estudio permitan el proceso de compra completo a través de Internet, puede traducirse en una pérdida de clientes potenciales, tomando en consideración la distancia que puede separar a los posibles esquiadores de las instalaciones.

Tabla 7

RESULTADOS DE LA EVALUACIÓN POR TIPO DE ESTACIÓN Y EN SU TOTALIDAD

\begin{tabular}{|l|l|c|c|c|c|c|c|c|c|}
\hline \multirow{2}{*}{ eMICA } & \multicolumn{2}{|c|}{$\begin{array}{c}\text { Esquí Al- } \\
\text { pino }\end{array}$} & \multicolumn{2}{|c|}{$\begin{array}{c}\text { Esquí } \\
\text { Nórdico }\end{array}$} & \multicolumn{2}{c|}{$\begin{array}{c}\text { Esquí In- } \\
\text { door }\end{array}$} & \multicolumn{2}{c|}{ Total 53 } \\
\hline \multirow{4}{*}{ Fase 1 } & Promoción & 0 & $0,0 \%$ & 9 & $42,8 \%$ & 0 & $0,0 \%$ & 9 & $17,0 \%$ \\
\cline { 2 - 11 } & Nivel 1 Información básica & 0 & $0,0 \%$ & 2 & $9,5 \%$ & 0 & $0,0 \%$ & 2 & $3,8 \%$ \\
\cline { 2 - 12 } & Nivel 2 Información rica & 0 & $0,0 \%$ & 7 & $3,3 \%$ & 0 & $0,0 \%$ & 7 & $13,2 \%$ \\
\hline \multirow{3}{*}{ Fase 2 } & Provisión & 24 & $77,4 \%$ & 11 & $52,4 \%$ & 1 & $100 \%$ & 36 & $67,9 \%$ \\
\cline { 2 - 11 } & Nivel 1 Interactividad baja & 0 & $0,0 \%$ & 2 & $9,5 \%$ & 0 & $0,0 \%$ & 2 & $3,8 \%$ \\
\cline { 2 - 11 } & Nivel 2 Interactividad media & 22 & $71,0 \%$ & 9 & $42,9 \%$ & 1 & $100 \%$ & 32 & $60,4 \%$ \\
\hline \multirow{2}{*}{ Fase 3 Interactividad alta } & 2 & $6,4 \%$ & 0 & $0,0 \%$ & 0 & $0,0 \%$ & 2 & $3,8 \%$ \\
\hline \multirow{2}{*}{ Proceso } & Total & 7 & $22,6 \%$ & 1 & $4,8 \%$ & 0 & $0,0 \%$ & 8 & $15,1 \%$ \\
\hline
\end{tabular}

Fuente: elaboración propia.

Por otro lado, si analizamos los resultados por tipo de estación, la modalidad «alpina» es la que acapara mayor número de sitios Web por niveles y fases, y es la que muestra unos resultados más positivos, explicado por el hecho de que es el tipo de estación más numerosa. Es de resaltar que el 22,6\% de las instalaciones se sitúa en la tercera fase, la inmensa mayoría se encuentra en el segundo nivel de la segunda fase (71\%) y que ninguna estación se encuentra ubicada en la primera fase.

Las estaciones de esquí «nórdico» no muestran un nivel tan elevado de adopción al comercio electrónico ya que un significativo $42,8 \%$ se sitúa en la primera fase, un $52,4 \%$ en la segunda y un escaso 4,8\% se ubica en la tercera fase. Por último, señalar que la única estación «Indoor» de nuestro país, se sitúa en el nivel medio de madurez, ya que está en el nivel 2 de la fase 2.

Dado que las estaciones de esquí Alpino son las más numerosas y las que mejores niveles de adopción alcanzan se ha procedido a comprobar si existía algún tipo de relación entre el tamaño de la estación y las aplicaciones comerciales de Internet. 
Tabla 8

RESULTADOS DE LA EVALUACIÓN POR TAMAÑO
DE LAS ESTACIONES DE ESQUÍ ALPINO

\begin{tabular}{|c|c|c|c|c|c|}
\hline eMICA & Estaciones de esquí Alpino & $\begin{array}{c}\text { Estaciones } \\
\text { Muy } \\
\text { Grandes }\end{array}$ & $\begin{array}{c}\text { Estaciones } \\
\text { Grandes }\end{array}$ & $\begin{array}{l}\text { Estaciones } \\
\text { medianas }\end{array}$ & $\begin{array}{c}\text { Estaciones } \\
\text { pequeñas }\end{array}$ \\
\hline Total & & 1 & 5 & 9 & 11 \\
\hline \multirow{3}{*}{ Fase 1} & Promoción & & & & \\
\hline & Nivel 1 Información básica & 2 & 5 & 9 & 11 \\
\hline & Nivel 2 Información rica & 2 & 5 & 9 & 11 \\
\hline \multirow{4}{*}{ Fase 2} & Provisión & & & & \\
\hline & Nivel 1 Interactividad baja & 2 & 4 & 7 & 7 \\
\hline & Nivel 2 Interactividad media & 1 & 1 & 2 & 4 \\
\hline & Nivel 3 Interactividad alta & 1 & 1 & 1 & 4 \\
\hline Fase 3 & Proceso & 1 & 1 & 1 & 4 \\
\hline
\end{tabular}

Fuente: elaboración propia.

La clasificación del tamaño de las estaciones se ha realizado por el parámetro denominado momento de potencia (MP). Es un parámetro definido por el STRMTG (Service Technique des Remontées Mécaniques et des Transports Guidés), organismo del gobierno de Francia encargado del registro y la seguridad de los remontes mecánicos de su país. El momento de potencia de una estación de esquí es la suma de los distintos MP de los remontes mecánicos que es el producto de su rendimiento por su desnivel. Mide la capacidad de hacer ganar altura a los pasajeros y se expresa en kilómetros/ esquiables /hora Definiremos los diferentes tamaños de una estación de la siguiente manera:

- Estaciones Pequeñas: Inferior a $2.500 \mathrm{Km}$-Esqs/h de MP acumulado.

- Estaciones Medianas: $2.500-6.000 \mathrm{Km}$-Esqs/h de MP acumulado.

- Estaciones Grandes: 6.000 - $15.000 \mathrm{Km}$-Esqs/h de MP acumulado.

- Estaciones Muy Grandes: superior a $15.000 \mathrm{Km}$-Esqs/h de MP acumulado.

De las 27 estaciones analizadas de la modalidad de esquí Alpino de España y Andorra clasificándolas por su tamaño mediante el MP por km esquiable, dos estaciones son consideradas muy grandes, cuatro grandes, diez medianas y once pequeñas. Fruto de la aplicación del modelo eMICA, observamos que siete estaciones de este tipo han alcanzado la fase 3 . Es un hecho remarcable que las estaciones que ocupan las dos últimas posiciones en la clasificación, es decir las de menor tamaño (Tavascán y Vall de Núria) son las que mejor han implementado elementos de comercio electrónico y mayor interactividad realizan. 
Tabla 9

CLASIFICACIÓN DE LAS ESTACIONES

DE ESQUÍ ALPINO POR TAMAÑO DE MP

\begin{tabular}{|c|c|c|c|}
\hline Posición & Estación & Momento de Potencia & Tamaño \\
\hline 1 & Grandvalira & 22.000 & Estación muy grande \\
\hline 2 & Baqueira Beret & 18.649 & Estación muy grande \\
\hline 3 & Vallnord & 14.000 & Estación grande \\
\hline 4 & Sierra Nevada & 13.375 & Estación grande \\
\hline 5 & Formigal & 8.662 & Estación grande \\
\hline 6 & Cerler & 7.273 & Estación grande \\
\hline 7 & La Molina & 5.935 & Estación mediana \\
\hline 8 & Astún & 4.687 & Estación mediana \\
\hline 9 & Masella & 4.665 & Estación mediana \\
\hline 10 & Candanchú & 3.978 & Estación mediana \\
\hline 11 & Port del Comte & 3.292 & Estación mediana \\
\hline 12 & Boí Taull & 3.130 & Estación mediana \\
\hline 13 & Panticosa & 2.782 & Estación mediana \\
\hline 14 & Port Ainé & 2.657 & Estación mediana \\
\hline 15 & Alto Campoo & 2.511 & Estación mediana \\
\hline 16 & Manzaneda & 2.511 & Estación mediana \\
\hline 17 & La Pinilla & 2.389 & Estación pequeña \\
\hline 18 & Valdezcaray & 2.169 & Estación pequeña \\
\hline 19 & Espot Esquí & 2.111 & Estación pequeña \\
\hline 20 & Navacerrada & 1.705 & Estación pequeña \\
\hline 21 & Fuentes de Invierno & 1.510 & Estación pequeña \\
\hline 22 & Valgrande-Pajares & 1.462 & Estación pequeña \\
\hline 23 & Vallter & 1.408 & Estación pequeña \\
\hline 24 & Javalambre & 1.293 & Estación pequeña \\
\hline 25 & Valdelinares & 1.087 & Estación pequeña \\
\hline 26 & Tavascán & 7.17 & Estación pequeña \\
\hline 27 & Vall de Núria & 457 & Estación pequeña \\
\hline
\end{tabular}

Fuente: elaboración propia a partir de www.nevasport.com (temporada 2014-2015).

Por lo que podemos concluir que no existe ningún tipo de relación entre el tamaño de la estación y las aplicaciones comerciales de Internet. Si nos centramos en los resultados por ubicación geográfica, los datos nos revelan que el grado de desarrollo de madurez 
es superior en el Pirineo al resto de sistemas. Podemos destacar el Pirineo Catalán, que sitúa a 4 estaciones en la tercera fase y 9 sitios en la fase 2. El Pirineo andorrano y el aragonés se colocan a continuación, ya que disponen de una estación cada zona en la fase 3 , y de dos y de nueve estaciones respectivamente en la segunda fase.

Tabla 10

CLASIFICACIÓN DE LA EVALUACIÓN

DE LAS ESTACIONES DE ESQUÍ ALPINO

\begin{tabular}{|c|c|c|c|c|}
\hline Posición & Tamaño & Clasificación & Estación & Tamaño \\
\hline 2 & 18649 & Fase 3 & Baqueira Beret & Estación muy grande \\
\hline 7 & 5935 & Fase 3 & La Molina & Estación grande \\
\hline 8 & 4687 & Fase 3 & Astún & Estación mediana \\
\hline 22 & 1462 & Fase 3 & Valgrande-Pajares & Estación pequeña \\
\hline 23 & 1408 & Fase 3 & Vallter & Estación pequeña \\
\hline 26 & 717 & Fase 3 & Tavascán & Estación pequeña \\
\hline 27 & 457 & Fase 3 & Vall de Núria & Estación pequeña \\
\hline
\end{tabular}

Fuente: elaboración propia.

Tabla 11

RESULTADOS DE LA EVALUACIÓN POR UBICACIÓN GEOGRÁFICA

\begin{tabular}{|l|c|c|c|c|c|c|c|c|c|c|c|c|c|c|}
\hline & $\begin{array}{c}\text { Pirineo } \\
\text { Catalán }\end{array}$ & $\%$ & $\begin{array}{c}\text { Pirineo } \\
\text { Aragonés }\end{array}$ & $\%$ & $\begin{array}{c}\text { Sistema } \\
\text { Ibérico }\end{array}$ & $\%$ & $\begin{array}{c}\text { Sistema } \\
\text { Central }\end{array}$ & $\begin{array}{c}\text { Cordillera } \\
\text { Cantábrica }\end{array}$ & $\%$ & Andorra & $\%$ & Indoor & $\%$ \\
\hline Fase 1 & 1 & 6,7 & 5 & 33,3 & 1 & 33,3 & & & & & 2 & 40,0 & \\
\hline Nivel 1 & 1 & 6,7 & 4 & 26,7 & & & & & & & 2 & 40,0 & & \\
\hline Nivel 2 & & & 1 & 6,7 & 1 & 33,3 & & & & & & & & \\
\hline Fase 2 & 10 & 66,7 & 9 & 60,0 & 2 & 66,7 & 5 & 100 & 5 & 83,3 & 2 & 40,0 & & \\
\hline Nivel 1 & 9 & 60,0 & 9 & 60,0 & 2 & 66,7 & 5 & 100 & 4 & 66,7 & 2 & 40,0 & & \\
\hline Nivel 2 & 1 & 6,7 & & & & & & & 1 & 16,7 & & & 1 & 100 \\
\hline Nivel 3 & & & & & & & & & & & & & & \\
\hline Fase 3 & 4 & 26,7 & 1 & 6,7 & & & & & 1 & 16,7 & 1 & 20,0 & & \\
\hline Total & 15 & & 15 & & 3 & & 5 & & 6 & & 5 & & 1 & \\
\hline
\end{tabular}

Fuente: elaboración propia.

A pesar de los resultados obtenidos y si comparamos nuestra investigación con la realizada por García-Lastra y Escalera (2008) cuyo trabajo de campo tuvo lugar en 2007, observamos que en los últimos 8 años se ha realizado un avance significativo. Las principales diferencias metodológicas con el estudio realizado en el 2007 se centran en 
la adaptación y actualización de algunos ítems que definen los diferentes niveles y en el número de sitios Webs analizados, 33 en el 2007 frente a 53 en la investigación actual.

Tabla 12

RESULTADOS COMPARADOS 2007-2015

\begin{tabular}{|c|c|c|c|c|c|c|c|c|c|}
\hline \multirow[t]{2}{*}{ eMICA } & & \multicolumn{2}{|c|}{ Esquí Alpino } & \multicolumn{2}{|c|}{ Esquí Nórdico } & \multicolumn{2}{|c|}{ Esquí Indoor } & \multicolumn{2}{|c|}{ Total } \\
\hline & & 2007 & 2015 & 2007 & 2015 & 2007 & 2015 & 2007 & 2015 \\
\hline \multirow{3}{*}{ Fase 1} & Promoción & $58,6 \%$ & $0,0 \%$ & $66,7 \%$ & $42,8 \%$ & $100 \%$ & $0,0 \%$ & $55,6 \%$ & $17,0 \%$ \\
\hline & Nivel 1 Información básica & $10,3 \%$ & $0,0 \%$ & $66,7 \%$ & $9,5 \%$ & $100 \%$ & $0,0 \%$ & $16,7 \%$ & $3,8 \%$ \\
\hline & Nivel 2 Información rica & $48,3 \%$ & $0,0 \%$ & - & $3,3 \%$ & - & $0,0 \%$ & $38,9 \%$ & $13,2 \%$ \\
\hline \multirow{4}{*}{ Fase 2} & Provisión & $41,4 \%$ & $77,4 \%$ & $33,3 \%$ & $52,4 \%$ & - & $100 \%$ & $36,1 \%$ & $67,9 \%$ \\
\hline & Nivel 1 Interactividad baja & $41,4 \%$ & $0,0 \%$ & $33,3 \%$ & $9,5 \%$ & - & $0,0 \%$ & $36,1 \%$ & $3,8 \%$ \\
\hline & Nivel 2 Interactividad media & - & $71,0 \%$ & - & $42,9 \%$ & - & $100 \%$ & - & $60,4 \%$ \\
\hline & Nivel 3 Interactividad alta & - & $6,4 \%$ & - & $0,0 \%$ & - & $0,0 \%$ & - & $3,8 \%$ \\
\hline Fase 3 & Proceso & - & $22,6 \%$ & - & $4,8 \%$ & - & $0,0 \%$ & - & $15,1 \%$ \\
\hline
\end{tabular}

Fuente: elaboración propia.

Los datos comparados confirman una mejora relevante en el grado de adopción en el comercio electrónico por parte de las estaciones de esquí y de montaña. Observamos que en el estudio de 2007 la gran mayoría de sitios Web estaban ubicados en la primera fase, sobre todo en las estaciones de esquí Nórdico. En esta investigación no se ubicó ningún sitio Web en la tercera fase.

Los resultados obtenidos en este apartado son coherentes con otros estudios a nivel nacional como por ejemplo en la «Encuesta de uso de TIC y Comercio Electrónico (CE) en las empresas 2013-2014», confeccionada por el INE. Según este informe el 98,3\% de las empresas poseen conexión a Internet y siete de cada diez disponen de sitio Web. Los servicios ofertados con mayor frecuencia en la página web de las empresas españolas son la presentación de la empresa, declaración de seguridad del sitio Web, el acceso a catálogos de productos o a listas de precios, y los menos frecuentes el seguimiento online de pedidos y la Personalización de la página Web para usuarios habituales o del diseño del producto. Resultados similares podemos encontrar en el estudio llevado a cabo por Fundetec (2014) de ámbito español.

\section{CONCLUSIONES}

El futuro del turismo en España en general y el de turismo de nieve y de montaña en particular deberá abordarse con nuevas perspectivas centradas en la innovación y en el aprovechamiento de las tecnologías de la información y de la comunicación como es el uso de Internet en todas sus dimensiones. No solo como elemento de promoción sino también como elemento de comercialización. 
Hemos comprobado a través de los resultados de la presenta investigación que actualmente, las estaciones de esquí son conscientes de la importancia de estar presentes en la Web, ya que todas ellas tienen acceso a Internet y disponen de sitio Web. Sin embargo, la mera presencia en la Red no es suficiente. Por lo que es necesario que estas organizaciones vayan más allá de la presencia tradicional, favoreciendo la interacción y colaboración en línea, la conectividad y la posibilidad de generar y compartir contenidos y conocimientos por parte de los usuarios, utilizando técnicas de la Web 2.0.

Los sitios Web de las estaciones de esquí en general, muestran un nivel de interactividad medio, en consonancia con su papel de destino turístico de primera magnitud. Sin embargo, únicamente la mitad de las Webs analizadas permitían el proceso de compra completo a través de Internet. La progresión más allá de este punto es probable que dependa de la función que se le otorgue a la presencia online, la comprensión de los beneficios que se derivan de las nuevas tecnologías a medida que estén disponibles, el nivel de innovación y de adopción de nuevas tecnologías dentro de la organización y la incorporación de funcionalidades Web 2.0 en forma de comentarios y valoraciones de los consumidores, la implementación de blogs, presencia en redes sociales y, por supuesto, de las limitaciones presupuestarias.

Los resultados del estudio sugieren que las estaciones de esquí y de montaña en España y Andorra se encuentran en una etapa relativamente avanzada de desarrollo y uso de Internet. Por tanto podemos afirmar que estas instalaciones están aprovechando las oportunidades que la Red presenta como una herramienta viable para la promoción de las zonas de montaña. Además, los resultados del estudio proporcionan una confirmación adicional de la utilidad del método por etapas para el desarrollo de sitios Web comerciales en el sector del Turismo propuesto por el modelo ampliado de Comercio de Internet (eMICA).

Podemos considerar las siguientes contribuciones principales fruto de este artículo:

1) El estudio establece un método válido para evaluar las etapas de desarrollo de sitios Web de estaciones de esquí y de montaña.

2) Proporciona un análisis de las distintas características de los sitios Web de las estaciones de esquí en función de su tipología y ubicación geográfica

3) Proporciona una evaluación en profundidad del estado de adopción del comercio electrónico en este sector de la industria turística que puede permitir una comparación interregional de sitios Web de estaciones de esquí.

Respecto a las limitaciones, el modelo eMICA tiene como principal desventaja que únicamente mide la presencia o ausencia de un servicio o aplicación, pero no mide la facilidad de encontrar un recurso o el tiempo para acceder a él, es decir, no evalúa la usabilidad de la página. También podemos añadir a lo comentado anteriormente, que debido a que el modelo eMICA responde a un proceso de adopción gradual de Internet, es posible encontrar casos en los cuales los sitios reúnan funcionalidades e incorporen elementos propios de diferentes etapas y niveles del modelo, por lo que en ocasiones es complejo tipificar el sitio Web analizado. Los servicios de los sitios Web de las estaciones de esquí están en constante cambio, lo cual implica que los resultados obtenidos 
pueden ir variando según el momento en que el estudio se lleve a cabo. Finalmente queremos señalar el hecho de que se ha estudiado las estaciones de esquí de un área geográfica en concreto.

Como futuras líneas de investigación, se propone ampliar el número de estaciones de esquí de otros países para disponer de más datos y poder comparar resultados. Además, se podría realizar entrevistas personales a los directivos de estas instalaciones, para identificar las principales barreras que encuentran al uso maduro de Internet que pueda justificar la escasa presencia de las estaciones de esquí en la tercera fase del modelo. Finalmente pretendemos complementar esta investigación con un análisis de contenidos Web para poder establecer mejor la situación de los sitios Web de este tipo de instalaciones así como su facilidad de uso.

\section{BIBLIOGRAFÍA}

ÁGUILA, A.R. DEL.; GARRIDO, A. y PADILLA, A. (2010): «Creación de valor online y redes sociales en el contexto del turismo cultural: El caso de los museos». Estudios Turísticos, $\mathrm{n}^{\mathrm{o}}$ 185, pp. 101-119.

ÁLVAREZ Y. (2014): La orientación al mercado en el sector turístico con el uso de las herramientas de la Web social, efectos en los resultados empresariales. Tesis Doctoral. Universidad de Cantabria. pp. 413.

ÁLVAREZ Y.; PÉREZ-GONZÁLEZ D. y SOLANA P. (2013): «Contribución de la Web 2.0 al desempeño organizacional en las empresas del sector turismo». Revista Puente, Revista Científica Universidad Pontificia Bolivariana Seccional Bucaramanga, vol. 17, $\mathrm{n}^{\mathrm{O}}$ 2. pp. 7-13.

BUHALIS, D. (2000); «Tourism and Information technologies: Past, present and future». Tourism Recreation Research, 25 (1), pp. 41-58.

BUHALIS, D. y LAW, R. (2008): «Progress in information technology and tourism management: 20 years on and 10 years after the Internet - The state of eTourism research». Tourism Management, vol. 29, nº 4, pp. 609-623.

BURGESS, L. y COOPER, J. (1998): «The Status of Internet Commerce in the Manufacturing Industry in Australia: A survey of Metal Fabrication Industries». Proceedings of the Second CollECTeR Conference on Electronic Commerce, pp. 65-73. Sydney.

BURGESS, L. y COOPER, J. (2000): «Extending the Viability of MICA (Model of Internet Commerce Adoption) as a Metric for Explaining the Process of Business Adoption of Internet Commerce». International Conference on Telecommunications and Electronic Commerce. Dallas (November).

BURGESS, L.; SARGENT, J. P.; COOPER, J. y CERPA, N. (2005): «A comparative analysis of the use of the Web for destination marketing by regional tourism organisations in Chile and the Asia Pacific». Collaborative Electronic Commerce Technology and Research. Chile: Universidad de Talca.

BURGESS, L.; PARRISH, B. y ALCOCK, C. (2011). «To what extent are regional tourism organisations (RTOs) in Australia leveraging the benefits of web technology for destination marketing and eCommerce?». Electronic Commerce Research, vol. 11 (3), pp. 341-355. 
CHUNG, Y. y BUHALIS, D. (2008): «Information needs in Online Social Networks». Information Technology Tourism, 10 (4), 267.

CRISTÓBAL, E.; DARIES, N. y MARTÍN, E. (2013). «El turismo y el comercio electrónico en España: un estudio del consumidor y de la empresa turística». Revista de Estudios Turísticos, $\mathrm{n}^{\circ}$ 195, pp. 79-99.

DARIES-RAMON, N.; CRISTOBAL-FRANSI, E. y MARTIN-FUENTES, E. (2014): «Promoción turística de las comunidades autónomas españolas a través de las redes sociales oficiales». Papers de Turisme, $\mathrm{n}^{\circ}$ 55, pp. 84-106.

DIPIETRO, R.B. y WANG, Y.R. (2010): «Key issues for ICT applications: impacts and implications for hospitality operations». Worldwide Hospitality and Tourism Themes, vol. 2, n ${ }^{\circ} 1$, pp. 49-67.

DOOLIN, B., BURGESS, L. y COOPER, J. (2002): «Evaluating the use of the Web for tourism marketing: A case study from New Zealand». Tourism Management, vol. 23 (5), pp. 557-561.

ESTEBAN, A: (1995): «Los nuevos desarrollos turísticos en España y su efecto en la recuperación y/o promoción económica». Papers de Turisme, $\mathrm{n}^{\circ}$ 17, pp. 33-44.

FALK, M. (2009): «Are multi-resort ski conglomerates more efficient?» Managerial and Decision Economics, vol. 30, pp. 529-538.

FALK, M. (2010): «A dynamic panel data analysis of snow depth and winter tourism». Tourism Management, vol. 31, pp. 912-924.

FUNDETEC (2014). Informe ePyme 2013. Análisis sectorial de implantación de las TIC en la pyme española. Documento disponible en: http://www.fundetec.es/publicaciones/ informe-epyme-2013/

GARCÍA-ABAD, A. (2012): «Estaciones de esquí, turismo y entorno rural de montaña: claves para una regulación de las estaciones de esquí ante el cambio climático». Acciones e Investigaciones Sociales, 31 (julio), pp. 91-135.

GARCÍA-LASTRA, O. y ESCALERA G. (2008): «El uso de los sitios Web en las Estaciones de Esquí y Montaña en España y Andorra». VII Congreso «Turismo y Tecnologías de la Información y las Comunicaciones» Turitec 2008, pp. 1-18.

GARCÍA-LASTRA, O. y ESCALERA G. (2009): «El sitio Web como herramienta de marketing en el sector turístico: el caso de las estaciones de esquí y montaña en Castilla y León». Boletín económico de Castilla y León, nº. 21, pp. 79-83

GERBAUX, F. y MARCELPOIL, E. (2006): «Gouvernance des stations de montagne en France: les spécificités du partenariat public-privé». La revue de géographie alpine, vol. 94 (1), pp. 9-19.

GONZÁLEZ-RODRIGO, E.; JIMÉNEZ-ZARCO, A.I. y TORRENT, J. (2010): «Usuarios de Internet y comercio electrónico en Turismo». Actas del VIII Congreso Turismo y Tecnologías de la Información y las Comunicaciones TuriTec. Málaga. pp. 1-17.

HJALAGER, A. (2010): «A review of innovation research in tourism». Tourism Management, Vol. 31, n 1, pp. 1-12.

HUERTAS, A. y MARINE-ROIG, E. (2015): «Destination brand communication through the social media: What contents trigger most reactions of users?». En I. Tussyadiah y A. Inversini (Eds.), Information and Communication Technologies in Tourism 2015), Cham, Switzerland: Springer, pp. 295-308. 
GONÇALVES, O. (2013): «Efficiency and productivity of French ski resorts». Tourism Management, vol. 36). pp. 650-657.

IET (2013): Informe anual 2012. Encuesta de Gasto Turístico (Egatur). Documento disponible en http://www.iet.tourspain.es.

IZQUIERDO, A.; MARTÍNEZ-RUIZ, M.P. y JIMÉNEZ ARCO, A.I. (2009): Análisis de las barreras en la compra de servicios turísticos por Internet: implicaciones para la gestión comercial en el sector. Observatorio (OBS*) Journal, 10. Disponible en

http://www.obs.obercom.pt/index.php/obs/article/view/306/282.

KIM, H. y FESENMAIER, D.R. (2008): «Persuasive Design of Destination Web Sites: An Analysis of First Impression». Journal of Travel Research, vol. 47 (august), pp. 3-13. INE (Instituto Nacional de Estadística) (2014): Encuesta sobre el uso de Tecnologías de la Información y las Comunicaciones (TIC) y del comercio electrónico en las empresas. Septiembre. Documento disponible en: http://www.ine.es/

LASANTA, T.; LAGUNA, M. y VICENTE-SERRANO, S.M (2007): «Do tourism-based ski resorts contribute to the homogeneous development of the Mediterranean mountains? A case study in the Central Spanish Pyrenees». Tourism Management, vol. 28, pp. 1326-1339

LAW, R. y BAI, B. (2008): «How Do the Preferences of Online Buyers and Browsers Differ on the Design and Content of Travel Websites?». International Journal of Contemporary Hospitality Management, vol. 20, nº 4, pp. 388-400.

LEUNG, D.; LAW, R.; VAN HOOF, H. y BUHALIS, D. (2013): «Social media in tourism and hospitality: A literature review». Journal of Travel \& Tourism Marketing, vol. 30(1-2), pp. 3-22.

LITVIN, S.; GOLDSMITH, R. y PAN, B. (2008): «Electronic Word-of-Mouth in Hospitality and Tourism Management». Tourism Management, vol. 29, pp. 458-468.

MARIMON, F.; VIDGEN, R.; BARNES, S.J. y CRISTÓBAL E. (2010): «Purchasing behaviour in an online supermarket: the applicability of E-S-QUAL». International Journal of Market Research, vol. 52 (1), pp. 111-129.

MATZLER, K. y SILLER, H.J. (2003): «Linking travel motivations with perceptions of destinations: the case of youth travelers in Alpine summer and winter tourism». Tourism Review, vol. 58 (4), pp. 6-11.

O'CONNOR, P. y FREW, A. (2000): «Evaluating electronic channels of distribution in the hotel sector: A Delphi study». Information Technology and Tourism, vol. 3 (3/4), pp. 177-193.

ORTEGA, F.J.; RÍOS, M.A.; RUIZ-JIMÉNEZ A. y CEBALLOS, C. (2012): «Cómo actuar de manera eficiente en la comercialización online del sector hotelero andaluz». Actas $V$ Jornadas de Investigación en Turismo: Turismo y Sostenibilidad. Sevilla, pp. 515-540.

PEARCE, P.L. (2005): Tourist behaviour: Themes and conceptual schemes. Clevedon: Channel View.

PÉREZ PÉREZ, M.; MARTÍNEZ SÁNCHEZ, A. y ABELLA, S. (2002): «El sector turístico y el comercio electrónico». Boletín Económico de Información Comercial Española, $\mathrm{n}^{\circ}$ 2.733. pp. 31-41.

PORTER, M. (2001): «Strategy and the Internet» . Harvard Business Review, vol. 79 (3), pp. 63-78. 
SAZ, M.I. y CARÚS, L. (2008): «La sostenibilidad del turismo recreativo de alta montaña». Cuadernos de Desarrollo Rural, vol. 5, nº 60, pp. 11-36.

SCHMIDT, S. (2006): Evaluación de los sitios Web de los hoteles e implicaciones para la gestión del marketing hotelero. Tesis doctoral. Islas Baleares: Universidad de las Islas Baleares.

TING, P.H.; WANG, S.T.; BAU, D.Y. y CHIANG, M.L. (2013): «Website evaluation of the top 100 hotels using advanced content analysis and eMICA model». Cornell Hospitality Quarterly, vol. 54 (3), pp. 284-293.

TSIMONIS, G. y DIMITRIADIS, S. (2014): «Brand strategies in social media». Marketing Intelligence \& Planning, vol. 32, pp. 328-344.

XIANG, Z. y GRETZEL, U. (2010): «Role of social media in online travel information search». Tourism Management, vol. 31 (2), pp. 179-188. 